\title{
Penalizing Physicians for Low-Value Care in Hospital Medicine: A Randomized Survey
}

\author{
Joshua M. Liao, MD, MSc 1,2,3*, Amol S. Navathe, MD, PhD $3,4,5$, Marilyn M. Schapira, MD, MPH ${ }^{3,5,6}$, \\ Arlene Weissman, $\mathrm{PhD}^{7}$, Nandita Mitra PhD ${ }^{3,8}$, David A. Asch, MD, MBA $3,4,5,6$
}

\begin{abstract}
${ }^{1}$ Department of Medicine, University of Washington, Seattle, Washington; ${ }^{2}$ UW Medicine Center for Scholarship in Patient Care Quality and Safety, University of Washington, Seattle, Washington; ${ }^{3}$ Leonard Davis Institute of Health Economics, University of Pennsylvania, Philadelphia, Pennsylvania; ${ }^{4}$ Department of Medical Ethics and Health Policy, University of Pennsylvania, Philadelphia, Pennsylvania; ${ }^{5}$ Center for Health Equity Research and Promotion, Philadelphia VA Medical Center, Philadelphia, Pennsylvania; ${ }^{6}$ Department of Medicine, University of Pennsylvania, Philadelphia, Pennsylvania; ${ }^{7}$ American College of Physicians, Philadelphia, Pennsylvania; ${ }^{8}$ Department of Biostatistics, Epidemiology and Informatics, University of Pennsylvania, Philadelphia, Pennsylvania.
\end{abstract}

Low-value services-those for which there is little to no benefit, little benefit relative to cost, or outsized potential harm compared with benefit-persist widely despite professional consensus, guidelines, and national campaigns to reduce them. As policy makers consider financially penalizing physicians to deter low-value services, physician support for such penalties remains unknown. We conducted a randomized survey experiment among physicians to evaluate how the framing of harms from low-value care-in terms of those to patients, healthcare institutions, or society-influenced physician support of financial penalties for low-value care services. Policy support rate was $39.6 \%$ overall and highest when the harms of low-value care were framed as costs to society (48.4\%). Compared with respondents receiving the "patient harm" version, those receiving the "societal harm" version (adjusted odds ratio [OR] 2.83; 95\% confidence interval [Cl], 1.20-6.69), but not the "institutional harm" framing (adjusted OR 1.53; 95\% Cl, 0.66-3.53), were more likely to report policy support. Our results suggest that emphasizing the impact of these harms may increase acceptability of financial penalties among physicians and contribute to the larger effort to decrease low-value care in hospital settings. Journal of Hospital Medicine 2018;13:4144. Published online first November 22, 2017. (C) 2018 Society of Hospital Medicine
$\mathrm{R}$ educing low-value care-services for which there is little to no benefit, little benefit relative to cost, or outsized potential harm compared with benefit-is an essential step toward maintaining or improving quality while lowering cost. Unfortunately, low-value services persist widely despite professional consensus, guidelines, and national campaigns aimed to reduce them. ${ }^{1-3}$ In turn, policy makers are beginning to consider financially penalizing physicians in order to deter low-value services. ${ }^{4,5}$ Physician support for such penalties remains unknown. In this study, we used a randomized survey experiment to evaluate how the framing of harms from low-value care-in terms of those to patients, healthcare institutions, or society-influenced physician support of financial penalties for low-value care services.

\section{METHODS}

\section{Study Sample}

By using a stratified random sample maintained by the American College of Physicians, we conducted a web-based survey

\footnotetext{
*Address for correspondence and reprint requests: Joshua M. Liao, MD, MSc, UWMC Health Sciences, BB 1240, 1959 NE Pacific Street, Seattle, WA 98195; Telephone: 206-616-6934; Fax: 206-616-1895; E-mail: joshliao@uw.edu

Additional Supporting Information may be found in the online version of this article.
}

Received: April 25, 2017; Revised: July 12, 2017; Accepted: August 5, 2017

2018 Society of Hospital Medicine DOI 10.12788/jhm.2879 among 484 physicians who were either internal medicine residents or internists practicing hospital medicine.

\section{Instrument Design and Administration}

Our study focused on 3 low-value services relevant to inpatient medicine: (1) placing, and leaving in, urinary catheters for urine output monitoring in noncritically ill patients; (2) ordering continuous telemetry monitoring for nonintensive care unit (nonICU) patients without a protocol governing continuation; and (3) prescribing stress ulcer prophylaxis for medical patients not at a high risk for gastrointestinal (GI) complications. Although the nature and trade-offs between costs, harms, and benefits vary by individual service, all 3 are promulgated through the Choosing Wisely ${ }^{\circledR}$ guidelines as low value based on existing data and professional consensus from the Society of Hospital Medicine. ${ }^{6}$

To evaluate intended behavior related to these 3 low-value services, respondents were first presented with 3 clinical vignettes focused on the care of patients hospitalized for pneumonia, congestive heart failure, and alcohol withdrawal, which were selected to reflect common inpatient medicine scenarios. Respondents were asked to use a 4-point scale (very likely to very unlikely) to estimate how likely they were to recommend various tests or treatments, including the low-value services noted above. Respondents who were "somewhat unlikely" and "very unlikely" to recommend low-value services were considered concordant with low-value care guidelines. 
TABLE. Physician Characteristics (by Survey Version)

\begin{tabular}{|c|c|c|c|c|c|}
\hline \multirow[b]{2}{*}{ Physician Characteristic } & \multirow[b]{2}{*}{$\begin{array}{c}\text { Overall } \\
(n=187)\end{array}$} & \multicolumn{4}{|c|}{ Version } \\
\hline & & $\begin{array}{l}\text { Patient } \\
(\mathrm{n}=60)\end{array}$ & $\begin{array}{l}\text { Societal } \\
(\mathrm{n}=62)\end{array}$ & $\begin{array}{l}\text { Institutional } \\
\quad(n=65)\end{array}$ & $P$ Value \\
\hline Age (median, IQR) & $39(33-47)$ & $39(35-44)$ & $42(33-48)$ & $39(33-47)$ & .85 \\
\hline Male (\%) & 57.8 & 58.3 & 53.2 & 61.5 & .85 \\
\hline Practicing physician & 87.2 & 86.7 & 87.1 & 87.7 & \\
\hline Resident physician & 12.8 & 13.3 & 12.9 & 12.3 & \\
\hline Practice incentives $(\%)^{a}$ & & & & & .71 \\
\hline Cost incentives & 20.9 & 25.0 & 22.6 & 15.4 & \\
\hline \multicolumn{6}{|l|}{ Attitudinal items (\% support) } \\
\hline $\begin{array}{l}\text { Providing financial incentives to individual physicians is an effective way to } \\
\text { improve the value of healthcare. }\end{array}$ & 73.3 & 76.7 & 75.8 & 67.7 & .45 \\
\hline $\begin{array}{l}\text { If a test or treatment has any chance of helping the patient, it is the clini- } \\
\text { cian's duty to offer it regardless of cost. }\end{array}$ & 70.1 & 78.3 & 69.4 & 63.1 & .18 \\
\hline $\begin{array}{l}\text { Clinicians should consider the costs of a test or treatment to society when } \\
\text { making clinical decisions. }\end{array}$ & 79.1 & 71.7 & 85.5 & 80.0 & .17 \\
\hline $\begin{array}{l}\text { Clinicians should consider the costs of a test or treatment to healthcare pro- } \\
\text { viders (practices, hospitals, and insurance companies) when making clinical } \\
\text { decisions for their patients. }\end{array}$ & 66.3 & 61.7 & 74.2 & 63.1 & .27 \\
\hline $\begin{array}{l}\text { It is inappropriate for anyone other than the treating clinician and patient to } \\
\text { decide if a test or treatment is "worth the cost." }\end{array}$ & 63.6 & 75.0 & 54.8 & 61.5 & .06 \\
\hline \multicolumn{6}{|l|}{ Concordance between intended behavior and low-value care guidelines (\%) } \\
\hline
\end{tabular}

Following the vignettes, respondents then used a 5-point scale (strongly agree to strongly disagree) to indicate their agreement with a policy that financially penalizes physicians for prescribing each service. Support was defined as "somewhat or strongly" agreeing with the policy. Respondents were randomized to receive 1 of 3 versions of this question (supplementary Appendix).

All versions stated that, "According to research and expert opinion, certain aspects of inpatient care provide little benefit to patients" and listed the 3 low-value services noted above. The "patient harm" version also described the harm of low-value care as costs to patients and risk for clinical harms and complications. The "societal harm" version described the harms as costs to society and utilization of limited healthcare resources. The "institutional harm" version described harms as costs to hospitals and insurers.

Other survey items were adapted from existing literature ${ }^{7-9}$ and evaluated respondent beliefs about the effectiveness of physician incentives in improving the value of care, as well as the appropriateness of including cost considerations in clinical decision-making.

The instrument was pilot tested among study team members and several independent internists affiliated with the University of Pennsylvania. After incorporating feedback into the final instrument, the web-based survey was distributed to eligible physicians via e-mail. Responses were anonymous and respondents received a $\$ 15$ gift card for participation. The protocol was reviewed and deemed exempt by the University of Pennsylvania Institutional Review Board.

\section{Statistical Analysis}

Respondent characteristics (sociodemographic, intended clinical behavior, and cost control attitudes) were described by using percentages for categorical variables and medians and interquartile ranges for continuous variables. Balance in respondent characteristics across survey versions was evaluated using $\chi^{2}$ and 
Kruskal-Wallis tests. Multivariable logistic regression, adjusted for characteristics in the Table, was used to evaluate the association between survey version and policy support. All tests of significance were 2-tailed with significance level alpha $=0.05$. Analyses were performed using STATA version 14.1 (StataCorp LLC, College Station, TX, http://www.stata.com).

\section{RESULTS}

Of 484 eligible respondents, 187 (39\%) completed the survey. Compared with nonrespondents, respondents were more likely to be female (30\% vs $26 \%, P=.001)$, older (mean age 41 vs 36 years, $P<.001$ ), and practicing clinicians rather than internal medicine residents ( $87 \%$ vs $69 \%, P<.001)$. Physician characteristics were similar across the 3 survey versions (Table). Most respondents agreed that financial incentives for individual physicians is an effective way to improve the value of healthcare (73.3\%) and that physicians should consider the costs of a test or treatment to society when making clinical decisions for patients (79.1\%). The majority also felt that clinicians have a duty to offer a test or treatment to a patient if it has any chance of helping them $(70.1 \%)$ and that it is inappropriate for anyone beyond the clinician and patient to decide if a test or treatment is "worth the cost" (63.6\%).

Concordance between intended behavior and low-value care guidelines ranged considerably (Figure). Only $11.8 \%$ reported behavior that was concordant with low-value care guidelines related to telemetric monitoring, whereas $57.8 \%$ and $78.6 \%$ reported concordant behavior for Gl ulcer prophylaxis and urinary catheter placement, respectively.

Overall, policy support rate was $39.6 \%$ and was the highest for the "societal harm" version (48.4\%), followed by the "institutional harm" (36.9\%) and "patient harm" (33.3\%) versions. Compared with respondents receiving the "patient harm" version, those receiving the "societal harm" version (adjusted odds ratio [OR] 2.83; 95\% confidence interval [Cl], 1.20-6.69), but not the "institutional harm" framing (adjusted OR 1.53; $95 \% \mathrm{Cl}, 0.66-3.53)$, were more likely to report policy support. Policy support was also higher among those who agreed that providing financial incentives to individual physicians is an effective way to improve the value of healthcare (adjusted OR $4.61 ; 95 \% \mathrm{Cl}, 1.80-11.80)$.

\section{DISCUSSION}

To our knowledge, this study is the first to prospectively evaluate physician support of financial penalties for low-value services relevant to hospital medicine. It has 2 main findings.

First, although overall policy support was relatively low (39.6\%), it varied significantly on the basis of how the harms of low-value care were framed. Support was highest in the "societal harm" version, suggesting that emphasizing these harms may increase acceptability of financial penalties among physicians and contribute to the larger effort to decrease low-value care in hospital settings. The comparatively low support for the "patient harm" version is somewhat surprising but may reflect variation in the nature of harm, benefit, and cost trade-offs for individual low-value services, as noted above, and physician

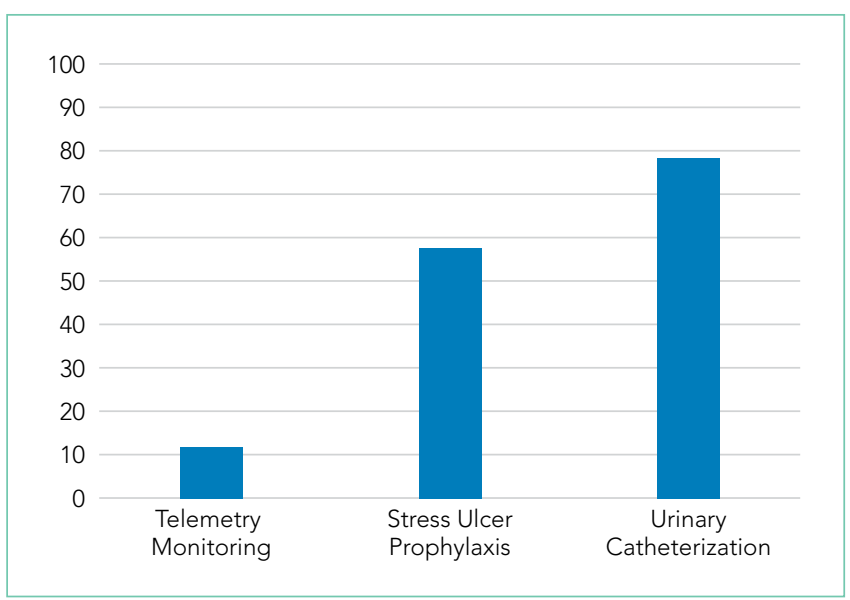

FIG. Percent Concordance between Intended Behavior and Low Value Care Guidelines.

belief that some low-value services do not in fact produce significant clinical harms.

For example, whereas evidence demonstrates that stress ulcer prophylaxis in non-ICU patients can harm patients through nosocomial infections and adverse drug effects, ${ }^{10,11}$ the clinical harms of telemetry are less obvious. Telemetry's low value derives more from its high cost relative to benefit, rather than its potential for clinical harm. ${ }^{6}$ The many paths to "low value" underscore the need to examine attitudes and uptake toward these services separately and may explain the wide range in concordance between intended clinical behavior and low-value care guidelines (11.8\% to $78.6 \%$ ).

Reinforcing policies could more effectively deter low-value care. For example, multiple forces, including Medicare payment reform and national accreditation policies, ${ }^{12,13}$ have converged to discourage low-value use of urinary catheters in hospitalized patients. In contrast, there has been little reinforcement beyond consensus guidelines to reduce low-value use of telemetric monitoring. Given questions about whether consensus methods alone can deter low-value care beyond obvious "low hanging fruit," 14 policy makers could coordinate policies to accelerate progress within other priority areas.

Broad policies should also be paired with local initiatives to influence physician behavior. For example, health systems have begun successfully leveraging the electronic medical record and utilizing behavioral economics principles to design interventions to reduce inappropriate overuse of antibiotics for upper respiratory infections in primary care clinics. ${ }^{15}$ Organizations are also redesigning care processes in response to resource utilization imperatives under ongoing value-based care payment reform. Care redesign and behavioral interventions embedded at the point of care can both help deter low-value services in inpatient settings.

Study limitations include a relatively low response rate, which limits generalizability. However, all 3 randomized groups were similar on measured characteristics, and experimental randomization reduces the nonresponse bias concerns accompanying descriptive surveys. Additionally, although we evaluat- 
ed intended clinical behavior in a national sample, our results may not reflect actual behavior among all physicians practicing hospital medicine. Future work could include assessments of actual or self-reported practices or examine additional factors, including site, years of practice, knowledge about guidelines, and other possible determinants of guideline-concordant behaviors.

Despite these limitations, our study provides important early evidence about physician support of financial penalties for low-value care relevant to hospital medicine. As policy makers design and organizational leaders implement financial incentive policies, this information can help increase their accept-

\section{References}

1. The MedPAC blog. Use of low-value care in Medicare is substantial. http:// www.medpac.gov/-blog-/medpacblog/2015/05/21/use-of-low-value-carein-medicare-is-substantial. Accessed September 18, 2017.

2. American Board of Internal Medicine Foundation. Choosing Wisely. http:// www.choosingwisely.org/. Accessed September 18, 2017.

3. Rosenberg A, Agiro A, Gottlieb M, etal. Early Trends Among Seven Recommendations From the Choosing Wisely Campaign. JAMA Intern Med. 2015;175(12): 1913-1920.

4. Centers for Medicare \& Medicaid Services. CMS Response to Public Comments on Non-Recommended PSA-Based Screening Measure. https://www.cms.gov/ Medicare/Quality-Initiatives-Patient-Assessment-Instruments/MMS/Downloads/eCOM-Development-and-Maintenance-for-Eligible-Professionals_CMS_ PSA_Response_Public-Comment.pdf. Accessed September 18, 2017.

5. Berwick DM. Avoiding overuse-the next quality frontier. Lancet. 2017;390(10090):102-104. doi: 10.1016/S0140-6736(16)32570-3.

6. Society of Hospital Medicine. Choosing Wisely. https://www.hospitalmedicine.org/choosingwisely. Accessed September 18, 2017.

7. Tilburt JC, Wynia MK, Sheeler RD, et al. Views of US Physicians About Controlling Health Care Costs. JAMA. 2013;310(4):380-388.

8. Ginsburg ME, Kravitz RL, Sandberg WA. A survey of physician attitudes and practices concerning cost-effectiveness in patient care. West J Med. 2000;173(6):309-394. ability among physicians and more effectively reduce low-value care within hospitals.

Disclosure: Drs. Liao, Schapira, Mitra, and Weissman have no conflicts to disclose. Dr. Navathe serves as advisor to Navvis and Company, Navigant Inc, Lynx Medical, Indegene Inc, and Sutherland Global Services and receives an honorarium from Elsevier Press, none of which have relationship to this manuscript. Dr. Asch is a partner and part owner of VAL Health, which has no relationship to this manuscript.

Funding: This work was supported by The Leonard Davis Institute of Health Economics at the University of Pennsylvania, which had no role in the study design, data collection, analysis, or interpretation of results.

9. Colla CH, Kinsella EA, Morden NE, Meyers DJ, Rosenthal MB, Sequist TD Physician perceptions of Choosing Wisely and drivers of overuse. Am J Manag Care. 2016;22(5):337-343.

10. Herzig SJ, Vaughn BP, Howell MD, Ngo LH, Marcantonio ER. Acid-suppressive medication use and the risk for nosocomial gastrointestinal tract bleeding. Arch Intern Med. 2011;171(11):991-997.

11. Pappas M, Jolly S, Vijan S. Defining Appropriate Use of Proton-Pump Inhibitors Among Medical Inpatients. J Gen Intern Med. 2016;31(4):364-371.

12. Centersfor Medicare \& Medicaid Services. CMS'Value-Based Programs. https://www.cms.gov/Medicare/Quality-Initiatives-Patient-Assessment-Instruments/Value-Based-Programs/Value-Based-Programs.html. Accessed September 18, 2017.

13. The Joint Commission. Requirements for the Catheter-Associated Urinary Tract Infections (CAUTI) National Patient Safety Goal for Hospitals. https:// www.jointcommission.org/assets/1/6/R3_Cauti_HAP.pdf. Accessed September 18, 2017

14. Beaudin-Seiler B, Ciarametaro M, Dubois R, Lee J, Fendrick AM. Reducing Low-Value Care. Health Affairs Blog. http://healthaffairs.org/ blog/2016/09/20/reducing-low-value-care/. Accessed September 18, 2017.

15. Meeker D, Linder JA, Fox CR, et al. Effect of Behavioral Interventions on Inappropriate Antibiotic Prescribing Among Primary Care Practices: A Randomized Clinical Trial. JAMA. 2016;315(6):562-570. 